\author{
Harold L. Atwood ${ }^{1}$ and \\ J. Martin Wojtowicz ${ }^{1}$ \\ Department of Physiology \\ Medical Sciences Building \\ University of Toronto \\ Toronto, Ontario M5S 1A8, Canada
}

\title{
Silent Synapses in Neural Plasticity: Current Evidence
}

Abstract

\section{Introduction}

POTENTIAL SIGNIFICANCE

OF SILENT SYNAPSES
Silent synapses, defined as structural specializations for neurotransmission that do not produce a physiological response in the receiving cell, may occur frequently in neural circuits. Their recruitment to physiological effectiveness may be an important component of circuit modification. In several nervous systems, evidence from electrophysiological and optophysiological measurements has established a strong case for the existence of silent synapses and for their emergence as active synapses with appropriate stimulation. During normal development and aging, synapses of individual neurons change in number, and many of these may be functionally silent at certain stages of their developmental trajectory. Changes in their status may contribute to shaping the properties of neural pathways during development, often in response to neural activity. In general, it is often difficult to distinguish physiological emergence of pre-established silent synapses from developmental maturation or de novo formation of new synapses. Several possible mechanisms for silent synapses and their recruitment are reviewed. These include incompletely assembled synapses that lack structural components, insufficient availability of key presynaptic proteins, and nonfunctional postsynaptic receptors, or presence of receptors that do not mediate a postsynaptic response except under specific conditions (conditionally silent synapses). The available silent synapses can often be rapidly activated, and conversely, active synapses appear to be rapidly silenced in many instances. These properties enable silent synapses to participate in short-term facilitation and depression. In addition, they may contribute to long-term facilitation and potentiation, especially during development.
The mammalian central nervous system (CNS) contains many millions of neurons, each of which characteristically receives thousands of individual inputs from other neurons and in turn provides thousands of outputs to other neurons. The sites of input and output are the synapses, which can be structurally defined with electron microscopy. Individual synapses vary enormously in their biochemical properties and physiological effects. The transmitter substances emitted by neurons at synapses, the receptors

\footnotetext{
${ }^{1}$ Corresponding authors.
}

LEARNING \& MEMORY 6:542-571 @ 1999 by Cold Spring Harbor Laboratory Press ISSN1072-0502/99 \$5.00

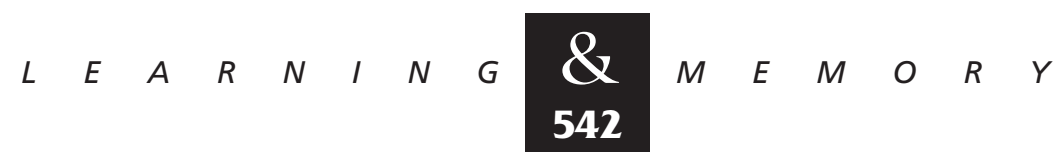


activated by the neurotransmitters, and the ensuing physiological responses (excitatory, inhibitory; facilitating, depressing; strong, weak; ionotropic, metabotropic) are all tremendously variable, and susceptible to modification by neural activity or during development.

A major question about neural synapses is as follows: Are all of the structurally defined synapses visible in electron micrographs physiologically potent? Since the early 1970 's, more and more evidence has accumulated that suggests that they are not. In particular, two early observations pointed to the occurrence of ineffective or incomplete (and thus, potentially silent) synapses. First, ultrastructural studies of crustacean motor neurons showed many synapses that appeared to lack docked (releasable) synaptic vesicles, and there were many more synapses than quanta released during neural activity (Jahromi and Atwood 1974). Secondly, and more dramatically, in the mammalian CNS, circumstances were found in which receptive fields could be suddenly expanded, implying the existence of newly awakened (previously silent) synapses (Wall 1977). Such observations indicated a strong possibility that many of the individual synapses formed between pairs of neurons are effectively silent, and do not produce a physiological effect when the presynaptic neuron is activated. It could even be that a majority of synapses in many neural pathways are physiologically silent, but such synapses might be recruited to physiological effectiveness with specific patterns of neural activity or through actions of neuromodulators and hormones. If so, this type of recruitment could be a major mechanism in pathway consolidation, learning, and memory. One can imagine that in many parts of the nervous system, there is a large reserve of silent synapses, which can be jolted into an active state by the right combination of stimuli, and can then enhance transmission either transiently or permanently in the neural circuits to which they contribute. According to this view, the nervous system is normally operating well below capacity, but has the potential for great enhancement and reconfiguration of local circuits. Silent synapses provide one mechanism (perhaps a major one) for enhanced performance of the nervous system.

The potential importance of silent synapses is a stimulus to review evidence supporting their existence and their possible functional significance. Evidence for silent synapses, particularly in the mammalian CNS, has been reviewed in several recent articles (Malenka and Nicoll 1997, 1999; Malenka 1998; Malinow 1998). We wish to extend the scope of the discussion to include other well-studied examples that offer relevant evidence and additional opportunities. New examples and evidence are appearing at an increasing rate.

First, we examine some basic features of synapses that might be involved in creation of silent synapses; then, we examine a selection of currently available evidence; and finally, we review briefly possible mechanisms and functional significance.

Synapses have both structural and functional attributes that are interrelated. Electron microscopy permits resolution of structural specialization at points of contact between nerve cells and their postsynaptic targets. The structural specialization is thought to reflect molecular features that impart the capability for fast release of neurotransmitter (fast exocytosis) from the presynaptic neuron during an

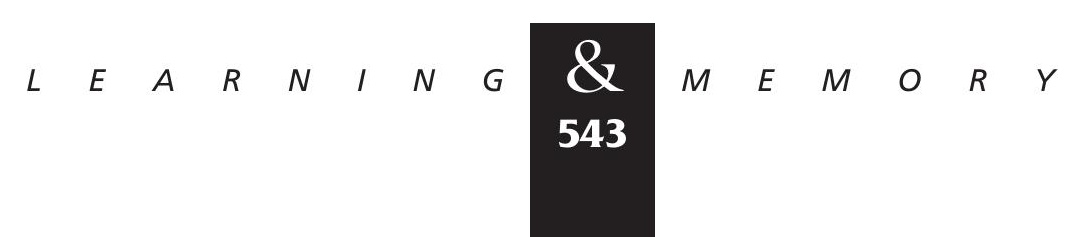


action potential. Most commonly, the structural definition of a synapse (Korn 1998) is taken to be the individual contact, comprising electron-dense pre- and postsynaptic membranes aligned rigidly with uniform (20-50 nm) separation (the synaptic cleft), and including variable specialized structures in both pre- and postsynaptic compartments. As illustrated in Figure 1, A and C, synaptic terminals or their varicosities often provide many individual synapses to a postsynaptic target cell, but in many cases, only a single synapse is present. In the literature, other uses of the term synapse appear; these have been reviewed recently by Korn (1998). The totality of synapses (which may be many) between one neuron and another, or between a neuron and a non-neural target, is commonly termed a synaptic connection (for review, see Faber et al. 1991).

Associated with the individual synapse's presynaptic membrane, dense projections often occur. Although their composition is not fully known, they appear to be focal points for accumulations of synaptic vesicles and are thus likely involved in directed docking and tethering of these vesicles, which are preferentially released at the margins of the presynaptic specializations in arthropod neuromuscular junctions (Fig. 1F). The presynaptic subregion involved in preferential release of neurotransmitter from synaptic vesicles is generally termed the active zone, from studies on the neuromuscular junction (del Castillo and Katz 1956; Couteaux 1970).

In many synapses of the CNS, particularly those on dendritic spines, the entire presynaptic face of the synapse may constitute an active zone, whereas in other cases, several active zones occur at one synapse (Jahromi and Atwood 1974; Cooper et al. 1995). Synaptic vesicles are preferentially released at the margins of an active zone, as shown in Figure $1 \mathrm{~F}$, or within it, in the case of many central synapses. Calcium channels are highly concentrated in the active zone (Fig. 1, D and E). Closely associated with calcium channels in this region are specialized molecules on the vesicles and on the presynaptic membrane, which form a multimolecular complex (core complex; Fig. 1B). The core complex, regulatory proteins, and calcium channel are thought to be mutually linked, and the entire ensemble is sometimes referred to as a secretosome (Bennett 1996). Regulatory molecules and calcium receptors are also present, generating complex molecular interactions (Wu et al. 1999). Only some of the relevant molecules are illustrated in Figure 1B. When this complex is activated by $\mathrm{Ca}^{2+}$, fast exocytosis ensues. Regulatory systems-calcium buffers, calcium sequestration, and extrusion mechanisms-profoundly affect synaptic strength. Postsynaptically, the membrane is specialized for localization of ligand-binding receptors, probably anchored to cytoskeletal components (Van Rossum and Hanisch 1999). Arrays of receptors are often seen in regular alignments in freeze-fracture replicas (Franzini-Armstrong 1976).

The structural components are linked to functional aspects of synaptic transmission. Synaptic vesicles contain and release transmitter substances in quantal events; voltage-activated calcium channels of the presynaptic active zone trigger impulse-evoked release when opened by nerve impulses; postsynaptic receptors bind released transmitter molecules and initiate a postsynaptic response, usually either movement of ions into the postsynaptic cell through an ion channel (ionotropic response), or alteration of a second-messenger pathway (metabotropic response).

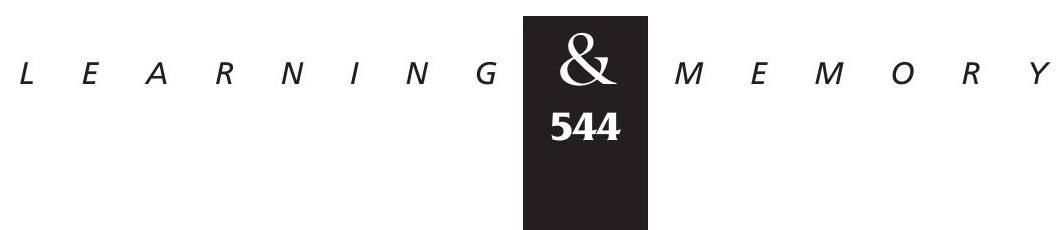



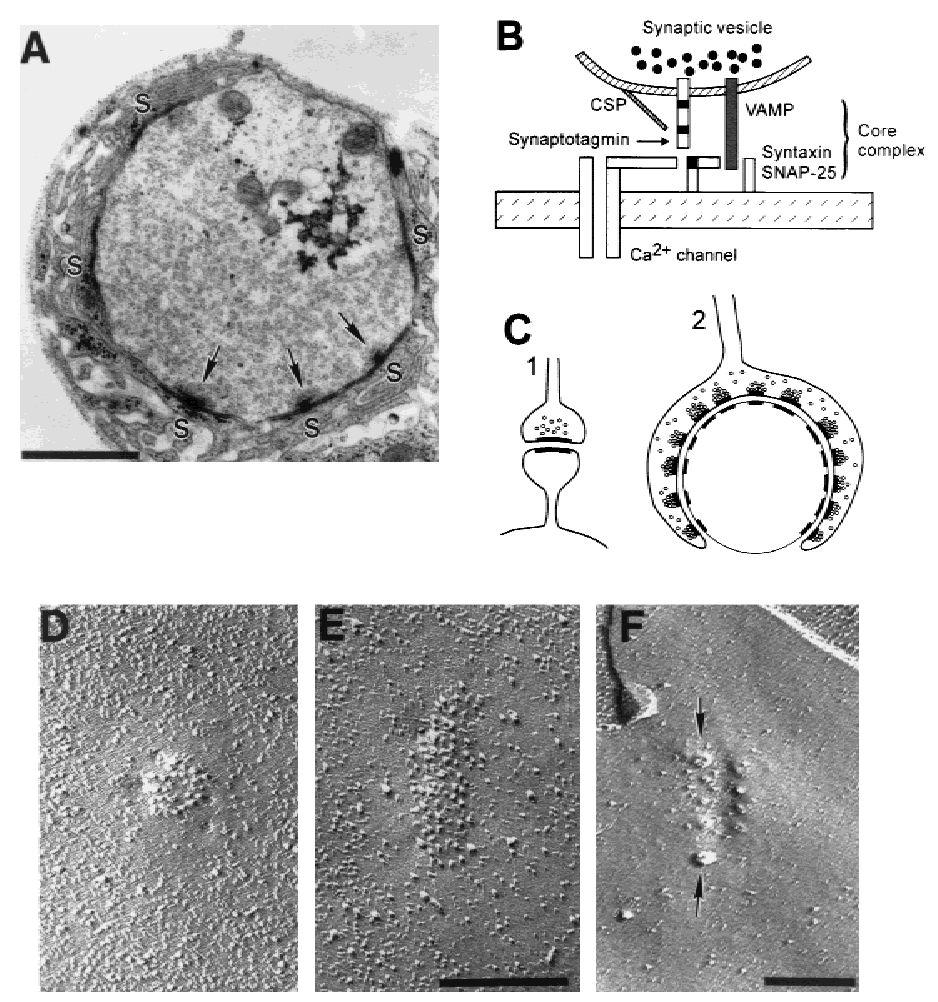

Figure 1: Structural features of synapses related to function. (A) Synapses of Drosophila neuromuscular junction, showing several synapses $(\mathrm{S})$ on one terminal. Characteristic structural features include the specialized electron-dense synaptic membranes, presynaptic dense bodies (T-shaped in Drosophila) located at active zones on the synapses (arrows), and specialized subsynaptic reticulum (postsynaptic side of the synapses). Synaptic vesicles are more densely clustered at dense bodies than elsewhere, and not all synapses possess dense bodies; note that in this section, only three of the six synapses show this structure. Scale bar, $1 \mu \mathrm{m}$. (B) Diagram of some of the essential molecular components of the presynaptic vesicular docking and fusion-promoting apparatus. The core complex (vesicular VAMP/synaptobrevin, and presynaptic SNAP-25 and syntaxin) is thought to be essential for fusion of vesicular and presynaptic membranes. Regulatory proteins, including synaptotagmin (a putative $\mathrm{Ca}^{2+}$ receptor) and cysteine string proteins that appear to affect $\mathrm{Ca}^{2+}$ sensitivity of release processes, modify the rate and calcium sensitivity of release. (Other known regulatory proteins are not shown in this diagram). Interaction of syntaxin with part of the presynaptic calcium channel (synprint site) is thought to enhance the coordination between calcium entry and vesicular fusion during fast exocytosis. $(C)$ Diagram of two types of mammalian synapse: (1) small synapse on a dendritic spine, with one active zone; (2) large caliciform somatic synaptic connection (calyx of Held), with many individual synapses and active zones. This structure has features in common with the arthropod neuromuscular junction $(A)$. The individual spine synaptic connection that frequently comprises a single synapse is typical of the majority of synapses in the mammalian CNS. $(D-F)$ Details of the synaptic active zone in freeze-fracture replicas (crayfish neuromuscular junction, inhibitory nerve terminal; after Govind et al. 1995). In $D$ and $E$, small and large active zones, respectively, exhibit collections of prominent intramembranous particles (putative calcium and calcium-activated $\mathrm{K}$ channels) in $p$-face views. Images of vesicular release (arrows), captured during chemical fixation, appear around the periphery of an active zone in an e-face view $(F)$. Scale bars, $0.2 \mu \mathrm{m}$. 
Modification of any of the synaptic components alters the physiological performance of the synapse, rendering it more or less effective.

Deficiencies or incomplete assembly of synaptic components can render the synapse silent (incapable of producing a functional response). Because deficiencies or lack of fine tuning may often occur at the molecular level, structural entities observed as synapses in electron micrographs may in fact be functionally silent, although ultrastructural clues to this situation may exist, as discussed below. Without additional critical evidence, one cannot accept counts of synapses in electron micrographs as being equivalent to the functional capabilities of the sampled network.

We envision several scenarios that could give rise to silent synapses (Fig. 2). A functional synapse would have all its normal structural components, and also possess an appropriate complement of the essential presynaptic molecules and postsynaptic receptors. Deficiencies in any of these components may affect the final operation of the synapse, rendering it physiologically silent. Thus, absence of, or interference with, any of several molecules of the presynaptic docking and fusion mechanisms (Fig. 2B) blocks synaptic transmission, as illustrated experimentally by studies of Drosopbila mutants (Littleton et al. 1993; Umbach et al. 1994) and injection of interfering peptides into nerve terminals (Bommert et al. 1993). Lack of functional receptors could also defeat successful neurotransmission (Fig. 2D). If the synapse is incompletely assembled, as could occur during development or senescence, it would not be able to

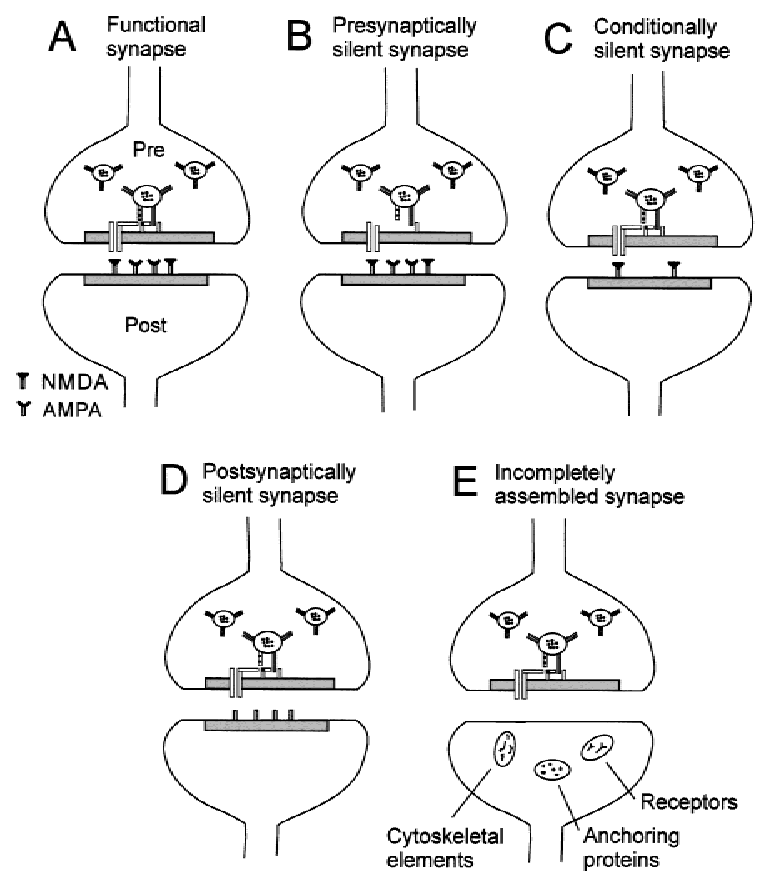

Figure 2: Possible types of silent synapse. (A) Complete synapse, possessing all of the necessary pre- and postsynaptic components. (B) Synapse silent due to presynaptic molecular deficiency. (C) Conditionally silent mammalian synapse (NMDA receptors only). (D) Synapse silent due to postsynaptic deficiency (receptors nonfunctional). ( $E$ ) Synapse in transition (postsynaptic components being assembled). 
perform physiologically (Fig. 2E), but could emerge into functionality either spontaneously or with a prodding stimulus.

A special case that occurs often in the mammalian CNS is that of synapses lacking functional AMPA-type glutamate receptors, but possessing NMDA-type glutamate receptors (Fig. 2C). Such synapses may be very prevalent early in development, and decrease in occurrence thereafter, as discussed below. Although these synapses are often referred to as silent synapses, we think they should be termed conditionally silent synapses, because they can express a physiological response when the membrane is depolarized, but not when it is hyperpolarized close to the resting potential (Fig. 3).

We conclude that two major criteria must be satisfied before one can claim the occurrence of silent synapses in a given neural pathway. First, synapses must be structurally present; second, they must be shown not to produce a physiological response under the conditions in which the system normally operates. Because conjoint physiological and structural observations at the level of individual synapses are difficult to obtain experimentally, it is not surprising that many claims of silent synapses are inferential rather than rigorously proven. Often, the claims are based on statistical analyses of physiological data that leave open more than one interpretation. Nevertheless, the accumulating evidence, incomplete or circumstantial as it may be, is compelling.
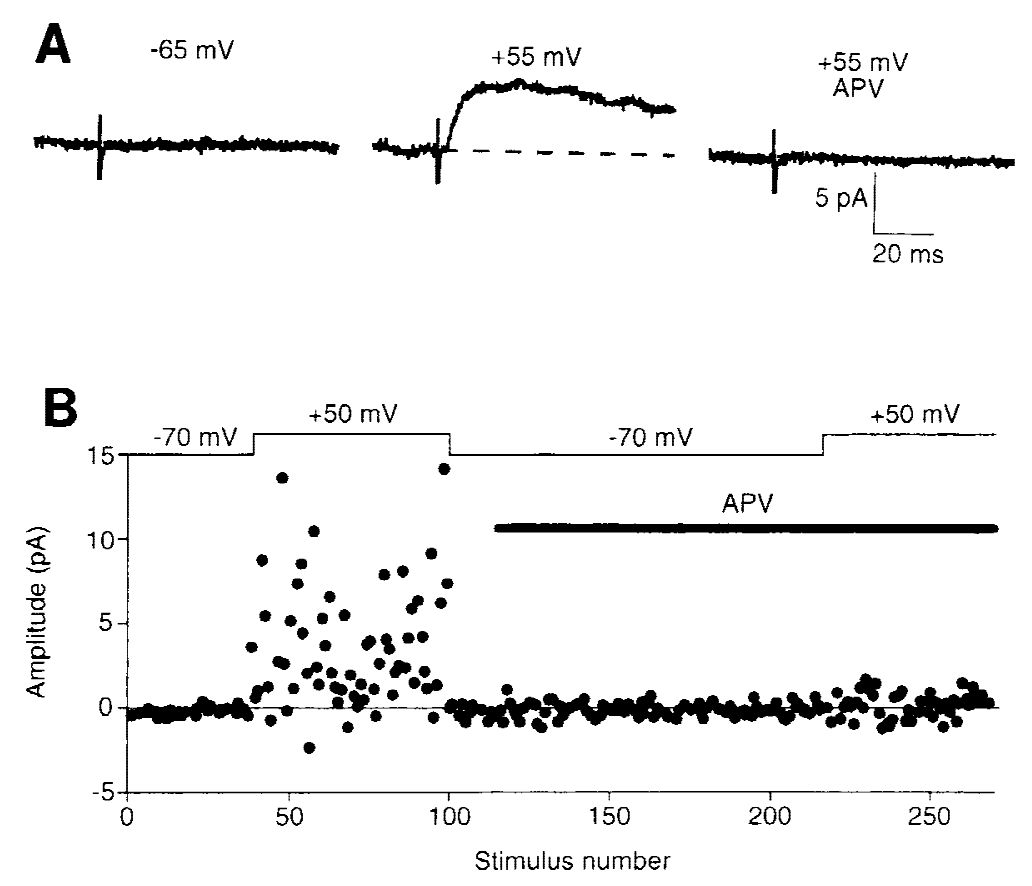

Figure 3: Conditionally silent synapses. Two experiments from mammalian CNS preparations to illustrate physiological participation of NMDA-only synapses as the membrane potential becomes depolarized. (A) EPSCs of synapses between neurons of CA3 and CA1 (rat hippocampus) appear at $+55 \mathrm{mV}$, but not at the resting membrane potential, and are blocked by APV (from Malinow 1998). (B) EPSCs observed when the membrane potential was changed from -70 to $+50 \mathrm{mV}$ (synapses between thalamic afferents and layer IV neurons in barrel-field cortex of rat). EPSCs were blocked by APV, indicating that NMDA receptors caused them (from Isaac et al. 1997). 
DEVELOPMENT

AND MATURITY
Of the studies on mammalian neurons in which the possibility of silent synapses has been raised, the majority have examined developmental processes, or tissue-cultured neurons that may develop differently from neurons in the intact CNS; studies of silent synapses in the mature nervous system are fewer in number. The general issue arises concerning the extent to which manifestations of silent synapses are attributable to a developmental sequence. Properties of long- and short-term plasticity differ in mature and immature synapses, and thus the age and developmental state of observed synapses is a significant variable.

Silent synapses have been implicated in brain plasticity of both young and mature animals. One could therefore ask whether silent synapses are more numerous in young, developing neurons or in mature ones? The dominant concept of the silent synapse in immature neurons of mammals (supported by many published studies) has been the synapse with NMDA-type glutamate receptors only, and lacking AMPA-type glutamate receptors (Fig. 2C). These receptors, due to their characteristic voltage and magnesium dependence, produce no postsynaptic current at the resting membrane potential even though release of the neurotransmitter (glutamate) occurs normally (Malenka and Nicoll 1997). There is convincing evidence for the occurrence of such silent synapses in the developing nervous system (Durand et al. 1996; Wu et al. 1996; Isaac et al. 1997; Rumpel et al. 1998; and many other recent studies). As maturation progresses, these silent synapses become rare and are presumably replaced progressively by active ones (possessing AMPA receptors). The transformation is thought to involve recruitment of AMPA-type glutamate receptors into the postsynaptic membrane (Shi et al. 1999). Activation of the NMDA receptors by glutamate with consequent inflow of $\mathrm{Ca}^{2+}$ is believed to be the major stimulus for recruitment of AMPA-type receptors.

There is only limited evidence for the presence of silent synapses in the mature mammalian brain. Immunogold labeling experiments suggest that $\sim 17 \%$ of synapses in the CA1 area of the hippocampus of mature rats and $28 \%$ in immature ones are devoid of a significant number of AMPA receptors (Nusser et al. 1998). It is not known whether the same synapses that lack AMPA receptors possess NMDA receptors, but if they do, they could be silent, under certain conditions. A few synapses with only NMDA receptor subunits have been reported (He et al. 1998). However, the NMDA-only silent synapses can only be considered conditionally silent, because on depolarization from the resting membrane potential, the synapses likely become instantly activated (Fig. 3). In the intact nervous system, such depolarization could occur through activation of adjacent excitatory AMPA synapses and, particularly in very young neurons, by means of depolarizing, GABA-dependent synapses (Ben Ari et al. 1997). Neurons in the intact brain normally have continuously fluctuating membrane potentials, and thus, NMDA-only synapses would be capable of producing a response much of the time. Moreover, even at rest, very young neurons are thought to possess relatively depolarized membrane potentials of around $-60 \mathrm{mV}$, which could unmask the NMDA-dependent depolarization even in the absence of additional synaptic input (Verheugen et al. 1999).

In summary, silent synapses possessing NMDA and not AMPA receptors, which appear to be much more frequent in the developing nervous system, are not likely to be truly silent. More exactly, they are 
LIFE SPAN OF A SYNAPSE conditionally silent. Use of the term silent synapse for conditionally silent synapses can lead inadvertently to a rather confusing description in which, for example, the observed bursting activity in a developing cortical circuit has been attributed to the activity of silent synapses (Golshani and Jones 1999). A more useful concept of the truly silent synapse is a more restricted one. For example, the classical Hebbian synapse, which is ineffective until a period of combined pre- and postsynaptic activity makes it active (Hebb 1949), would fit the stricter definition. According to this concept, the NMDA receptor can be a suitable trigger for the associative strengthening of such synapses by the right combination of pre- and postsynaptic mechanisms.

In relation to the question of synaptic development, the life span of individual synapses is relevant. Observations on several neuronal systems suggest that synapses may form and turn over rapidly in some cases For example, molluscan and leech neurons can apparently form new synapses within minutes when brought together in culture (Haydon 1988), and synapses in the optic system of insects undergo very rapid (sometimes diurnal) changes in size and number (Meinertzhagen 1993). In contrast, mammalian neuromuscular junctions, once established, remain in place with minor morphological alterations for a major portion of the lifetime of the animal (Balice-Gordon and Lichtman 1990). Conditions in culture differ from those in the intact brain, and the time course of individual synapse formation or dissolution is not well understood in the latter case. Published accounts indicate that synaptic age is variable and system or context specific.

In the mammalian CNS, the lifetime of a synapse is not well established. If silent synapses exist and if they are activated by activity to form a memory trace in the brain, one may wonder how long such a trace (if dependent on individual synapses) can last? Whereas synaptic connections in mature CNS are generally stable and maintained by homeostatic activity-dependent mechanisms (Turrigiano 1999), they can be potentiated for several weeks in the intact brain (Bliss and Lomo 1973). However, it is not clear if the individual synapses comprising the total synaptic connection can last that long, so the question of the synaptic turnover arises.

In some known systems, synapses can be formed continually during the lifetime of the animal. An example from the mammalian CNS concerns the generation of new neurons in the dentate gyrus of the hippocampus (Fig. 4). Neurons giving rise to the perforant pathway that enters the hippocampal formation are thought to continuously form new synaptic junctions, even in the adult brain (Wang et al. 2000). This results from production of new neurons (adult neurogenesis) within the dentate gyrus, a target of the perforant pathway. Thus, existing terminals of the perforant pathway probably sprout and form new synaptic junctions on newborn neurons. The physiological properties of these new synapses are different from those on mature neurons (Wojtowicz 1998). It is not known how long such synapses last, but some turnover likely occurs; as granule cells age and lose dendritic branches, synapses must atrophy as well (Fig. 4). In the rat, mature granule neurons with sparse dendritic arborization can be observed in animals that are 1-2 months old (J.M. Wojtowicz, unpubl.). These could be cells whose dendrites are being 

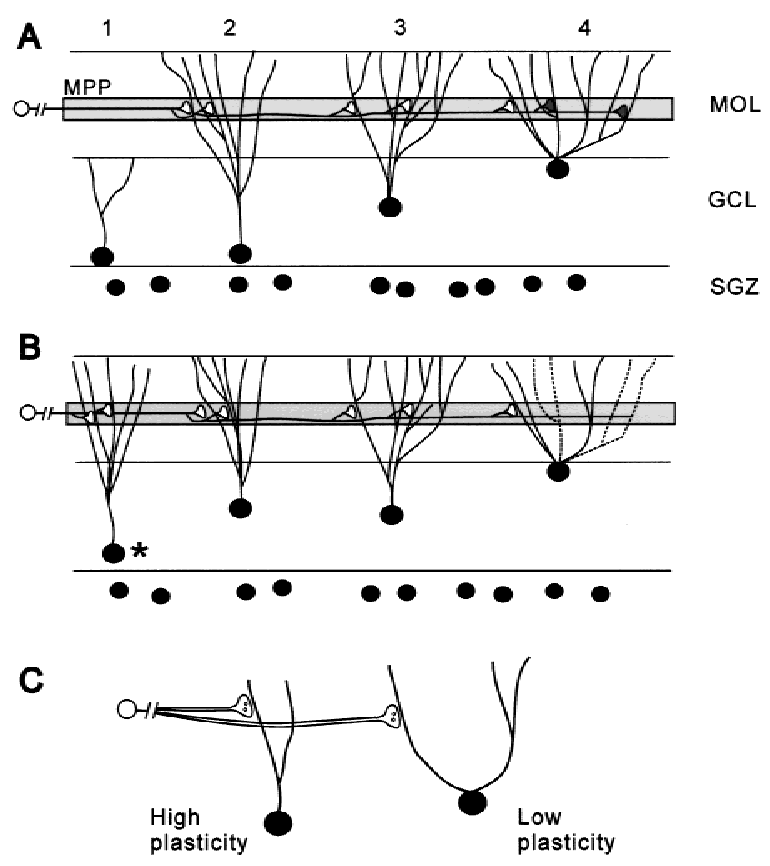

Figure 4: Synaptic turnover in the mammalian dentate gyrus. New neurons (1) are generated in the granule cell layer $(\mathrm{GCL})$ throughout life $(A, B)$. The new neurons grow dendrites that enlarge as the neuronal cell body migrates away from the hilus $(2,3)$. Synapses $(\bigcirc)$ formed by the medial perforant path (MPP) afferents on new neurons $(*)$ are younger than those already established on neurons that matured earlier. As neurons mature, some dendrites retract, degenerate, or are remodeled (4), and synapses are lost from such dendrites $(\mathbf{O})$. (MOL) Molecular layer; (SGZ) subgranular zone, located between GCL and hilus. (C) The existing synapses in young and mature neurons possess high or low capacity for plasticity respectively.

phased out. Such cells may eventually be replaced by newly produced ones.

Another well-studied case in which synaptic turnover occurs throughout life is the excitatory (glutamatergic) motor neuron of crustaceans, which has many morphological features in common with central neurons (Atwood and Wojtowicz 1986). In the lobster, early stages of innervation have been documented, and the same identified neuron can be sampled in animals of different age. The neuron continues to grow throughout the life of the animal (50 years or more), creating new branches to keep up with muscle growth (Atwood and Govind 1990). Synapses are continuously replaced, and new ones formed, as growth occurs (Fig. 5). The same neuron thus possesses synapses of different age. The most distal varicosities on a nerve branch have lower quantal content, but just as many ultrastructurally defined synapses, as the more proximal varicosities, suggesting a higher proportion of silent synapses on younger varicosities (Cooper et al. 1995).

The occurrence of synapses of different age on a single neuron, or within a population of neurons, is likely to affect the prevalence of silent synapses. Developing neurons or neuronal branches probably have a higher incidence of silent synapses. Synaptic generation or senescence creates synapses in which one or more of the underlying causes of 


\section{Manifestations and Evidence}

CRUSTACEAN

NEUROMUSCULAR SYSTEM
The initial impetus for the hypothesis that a substantial complement of silent synapses exists on crustacean glutamatergic motor nerve terminals was provided by three-dimensional ultrastructural reconstructions of synaptic boutons (Jahromi and Atwood 1974), on which a mixed population of synapses was observed: Most had one or two presynaptic dense bodies, but some (often $20 \%$ ) had none (see Fig. 1A), and, in addition, lacked the characteristic clusters of docked synaptic vesicles associated with the dense body. These synapses were postulated to be incomplete, and functionally silent. The same pattern has been found also for Drosophila motor nerve terminals (Atwood et al. 1993).

Additional evidence for synaptic heterogeneity has arisen from statistical studies of transmitter release. Quantal analyses based on simple or compound binomial models, in which parameters $n$ (number of responding units) and $p$ (average probability of unit response) determine overall quantal content $m$ (number of quantal units released per impulse; $m=n p$ ), were applied to the crayfish nerve terminal (Johnson and 
Wernig 1971; Zucker 1973; Smith et al. 1991; Wojtowicz et al. 1991). The general finding, for tonic motor axons, has been that quantal content is characteristically low ( 2 or less) at low frequencies of stimulation, and values for $n$ are also low (typically 2 or 3 ). In contrast, reconstructed boutons usually have 20-40 individual synapses (Wojtowicz et al. 1994; Cooper et al. 1995). If the value of $n$ represents synapses that can release transmitter, as suggested initially by Zucker (1973), there is a large excess of morphological synapses over functional ones. This is true even if synapses lacking a presynaptic dense body are excluded. At higher frequencies of stimulation, facilitation of transmitter release occurs ( $m$ increases). Both $n$ and $p$ increase with frequency (Wojtowicz et al. 1994), suggesting addition of active synapses.

Statistical procedures used to analyze transmitter release cannot in themselves distinguish between restriction of release to $n$ synapses, and distribution of release over many synapses, with $n$ representing the mean number of synapses primed for release (Worden et al. 1997).

Activity-dependent uptake of fluorescent dyes in synaptic vesicles [in particular, FM1-43 (Angleson and Betz 1997)] provides a distinguishing test. If release is distributed over all synapses, bright spots should occur over the bouton wherever there are synapses. However, in several tests (Quigley et al. 1999), crustacean tonic boutons develop relatively few bright spots, and much of the bouton's surface lacks focal labeling (Fig. 6A). Reconstructed boutons, especially the larger ones, invariably show more synapses than bright spots (Fig. 6C,D). The bright spots are larger than synapses and may represent several, with merged vesicle clusters. Nevertheless, it is clear that release probability is not uniformly distributed among synapses on a bouton, and that there is likely a substantial number of silent synapses.

In correspondence with the statistical results that have shown an increase in binomial parameter $n$ at higher frequencies, the number of bright spots on terminals also increases when the frequency is increased (Fig. 6B). The interpretation of this result is that some synapses that were not active at the lower frequency are stimulated to participate in exocytosis and vesicle retrieval at the higher frequency. Increased residual $\mathrm{Ca}^{2+}$ is likely to play a role in recruitment of initially silent synapses during frequency facilitation (Tank et al. 1995).

An additional study on crayfish terminals undergoing facilitation of release with application of serotonin used double labeling, first with FM1-43, and then, after serotonin application, with another dye, FM4-64, emitting at a longer wavelength (Wang and Zucker 1998). New synapses recruited with serotonin would be expected to fluoresce at the longer wavelength. Although a few such spots appeared, the general result was that those stained with FM1-43 prior to serotonin application became brighter and larger. Given the resolution available with the fluorescence microscope, it was not possible to distinguish between enlargement of the releasable pool of synaptic vesicles, and recruitment of previously silent synapses.

Long-term facilitation (LTF), induced by a train of stimulation at $10 \mathrm{~Hz}$ or higher, enhances synaptic transmission of crustacean tonic motor neurons for hours (Sherman and Atwood 1971). The potentiation has been shown to be presynaptic in origin (Wojtowicz and Atwood 1985), and is similar in many respects to that described for long-term potentiation (LTP) in the mammalian mossy fibers in the hippocampus,

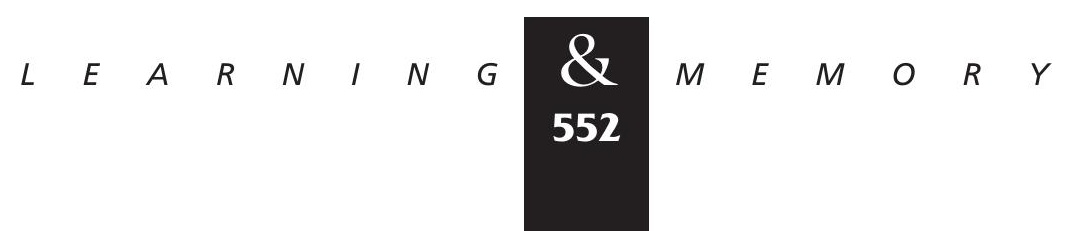


A

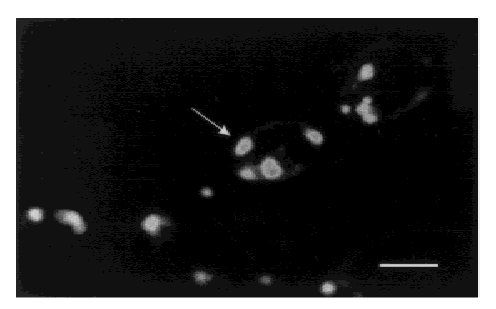

B

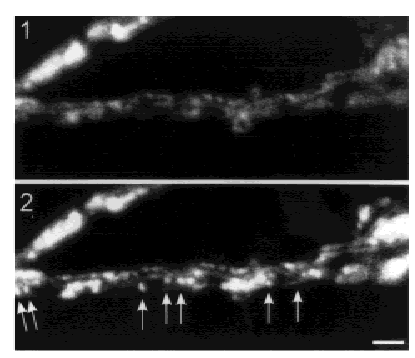

C

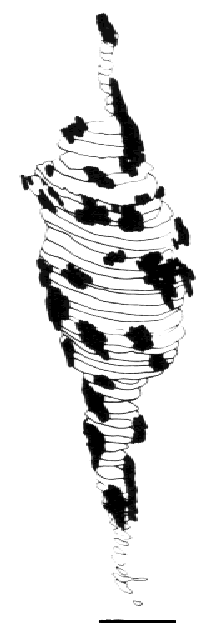

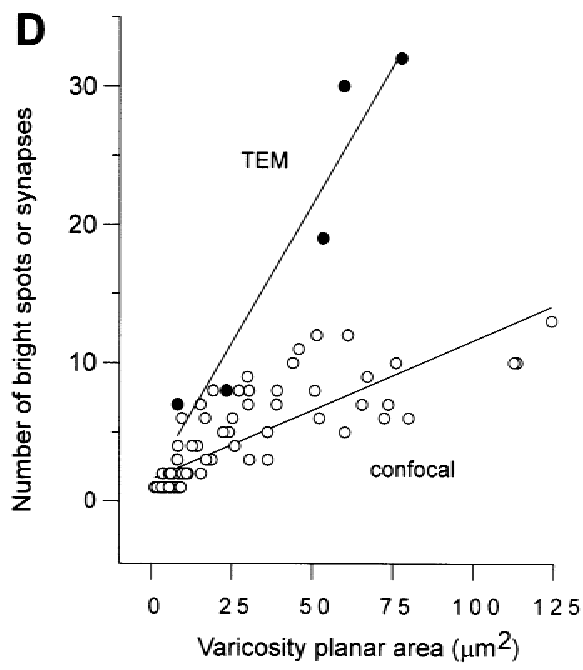

Figure 6: Visible evidence for silent synapses. (A) Boutons of a crustacean motor axon stimulated in the fluorescent dye FM1-43 develop several prominent bright spots (arrow) per bouton, each spot indicating one or more active synapses. Smaller boutons have fewer bright spots. (B) Stimulation of axons of the crayfish opener muscle at a relatively low frequency (5 Hz in 1) produces bright spots along the terminal. Increasing the frequency to $15 \mathrm{~Hz}$ (2) induces additional bright spots (some indicated by arrows) that were not visible at the lower frequency; in addition, preexisting spots became brighter and often larger. $(C)$ Reconstruction of a bouton from serial electron micrographs typically reveals $\sim 20-40$ individual synapses. $(D)$ The number of individual synapses found on reconstructed boutons (TEM) is usually greater than the number of bright spots appearing on boutons of the same size after stimulation in FM1-43 (confocal), especially for larger boutons $(A, B, D$, modified from Quigley et al. 1999; C modified from Cooper et al. 1996).

although the underlying mechanisms differ in some respects. For crustacean LTF, consistent results were obtained from statistical analysis: $n$ increased but not $p$, and increased $n$ could account for the observed potentiation (Wojtowicz et al. 1994). The result differs from that described above for frequency facilitation, suggesting that the number of responding units increased after LTF induction, whereas overall probability of release for participating synapses was unaltered. Although optical measurements with FM1-43 have not been attempted for LTF, electron microscopic surveys indicated a small but significant increase in complex synapses (those with multiple active zones, thought to have a enhanced probability of release). Thus, there is partial evidence for a structural shift in the synapse population (possibly due to rearrangement 
VERTEBRATE

NEUROMUSCULAR JUNCTIONS or splitting of active zones) which would favor a larger number of participating synapses, and a reduced number of silent synapses (Wojtowicz et al. 1994).

Collectively, the results for crustacean terminals support recruitment of pre-existing silent synapses to an active state in both short-term (frequency) facilitation, and LTF. In both cases, presynaptic mechanisms are responsible.

Neuromuscular junctions on skeletal muscles of vertebrates are typically organized to release a large amount of neurotransmitter (many quantal units) for each impulse. Nevertheless, on different muscle fibers, the effectiveness of transmission differs greatly, and this cannot be accounted for on the basis of differences in active zone (calcium channel) particles (Pawson et al. 1998). By implication, presynaptic factors in addition to structural ones regulate the probability of transmitter release at active zones of different junctions, as in crustaceans. Within junctions labeled by FM1-43, hot spots can be distinguished at which the rate of dye uptake and turnover is much higher than elsewhere (Betz et al. 1992). Physiological recordings also indicate hot spots of transmitter release along the junction (Wernig 1976; Macleod et al. 1999). A clear parallel exists with the crustacean junction, in which individual synapses are separated rather than consolidated into a continuous junction.

Varicosities of sympathetic nerve endings on smooth muscle characteristically have a low probability of release individually (Lavidis and Bennett 1992; Brain et al. 1997). Many of them are essentially physiologically silent. Antibody labeling of the varicosities for presynaptic proteins (syntaxin and proteoglycan SV2) suggested active zones, but after induced activity, individual active zones differed greatly in antibody labeling for the amino-terminal sequence of synaptotagmin, which is exposed during vesicle exocytosis. The labeling procedure indicated large differences in synaptotagmin endowment among varicosities, likely contributing to their different capabilities for exocytosis. Thus, differences in presynaptic proteins are a factor influencing synaptic potency.

Nervous systems and individual neurons of gastropod molluscs (especially Aplysia) have been extensively investigated; activity-dependent changes in neuronal morphology and synaptic plasticity have been described, and underlying mechanisms involving both short-term modulation of synaptic strength and long-term modification of synaptic connections through transcriptional regulation of several key molecules have been elucidated (Kandel and Schwartz 1982; Abel and Kandel 1998). This extensive work does not often address the issue of silent synapses, partly because optical and electrophysiological observations at the level of individual synapses are difficult to obtain in the intact molluscan nervous system.

Nevertheless, evidence for rapid changes in the population of responding synapses has been obtained for synaptic connections between sensory and motor neurons in tissue culture (Coulson and Klein 1997; Royer et al. 1999). Synaptic potentials showing rapid depression characteristically exhibit changes in $n$ but not in $p$, when subjected to quantal analysis (Fig. 7). An explanation for this finding is that part of the initially responding population of synapses rapidly becomes inactive (silent). These changes are reversed by serotonin, indicating rapid modulation of synaptic 
A

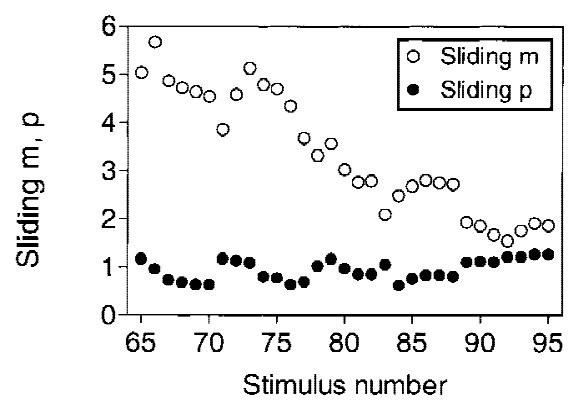

B

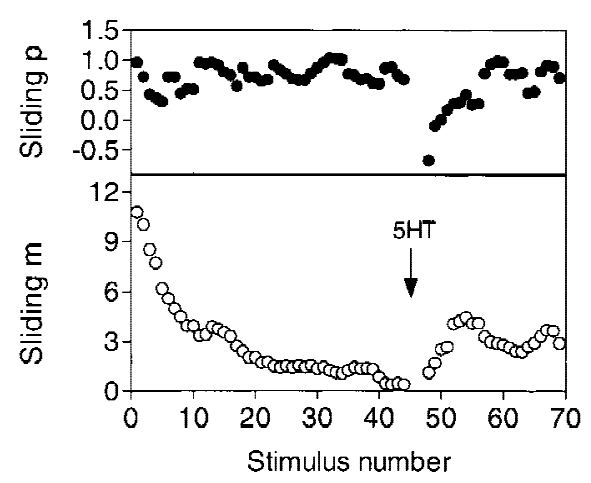

Figure 7: Statistical evidence for silent synapses. Analysis of EPSCs during trains of stimuli indicates progressive loss of contributing units (postulated to be individual synapses). Application of serotonin (5-HT) after depression increases the estimated number of responding units (synapses). (A) Absence of change in $p$ with homosynaptic depression at Aplysia sensory neuron-motor neuron synapse in culture. The quantal content $(m)$ and probability of release $(p)$ were estimated from the variance and mean of groups of five consecutive evoked EPSPs, moving one EPSP at a time; these estimates are called sliding $m(\bigcirc)$ and sliding $p(\mathbf{O})$, respectively. Despite the severalfold decrease in the quantal content, $p$ remains fairly constant. $(B)$ Estimated changes in $m$ and $p$ with homosynaptic depression and serotonin-induced facilitation at Aplysia sensory neuronmotor neuron synapse in culture. During repetitive stimulation, estimated quantal content and sliding $m$ declined, but increased with application of serotonin, whereas sliding $p$ showed little change except at the time of serotonin application. Data suggest that 5-HT and depression alter $m$ by changing the number of active synapses. Details of statistical procedures are given in Royer et al. (1999). (Courtesy of Dr. Marc Klein, University of Montreal, Montreal, Canada).

FISH NERVOUS SYSTEM

transmission leading to recruitment of some of the silenced synapses. Thus, statistical evidence in this system indicates an important role for silent synapses in short-term plasticity; but at present, corresponding optical or ultrastructural evidence is not available.

Similar rapid changes in depression occur in crustacean neurons (Bruner and Kennedy 1970) and in the mammalian CNS (Stricker et al. 1998); in both cases, rapid silencing of synapses remains a possible mechanism, but other explanations cannot yet be excluded.

Two types of silent synaptic connections have been found in the Mauthner cell of the fish. Two afferent systems make synaptic connections on this identifiable giant neuron; one set of afferents (from interneurons) is glycinergic and inhibitory, whereas a second set (club endings of eighth nerve primary afferents) is excitatory, providing electrical and chemical synapses conjointly to the Mauthner cell (Faber et al. 1991).

Some of the inhibitory glycinergic connections between interneurons and the Mauthner cell were ineffective, but could be made functional by high-frequency trains of stimuli and by conjoint stimulation of two parallel converging inputs (Charpier et al. 1995). Previously silent connections produced a response when additional connections were active. The effect was attributed to lateral interaction of transmitter released by the silent neuron onto receptors of the adjacent active synapses (transmitter 


\section{MAMMALIAN CNS}

EVIDENCE FROM

ELECTROPHYSIOLOGICAL AND

OPTOPHYSIOLOGICAL EXPERIMENTS overflow), implying that the receptors associated with the silent neuron were ineffective (Fig. 2D).

Excitatory inputs from club endings were often observed to lack the chemical component of transmission. This was in contrast to the electrical synapses of the same inputs, which were always effective, and served as an indicator for the arrival of the afferent impulse at the presynaptic ending. Each ending contains multiple active zones, yet there is frequently no transmitter release unless high-frequency stimulation is given, or the action potential broadened by presynaptic injection of cesium chloride. The evidence indicates that silence of this excitatory chemical synapse is presynaptic in origin (Faber et al. 1991).

The observations from the Mauthner cell provide convincing physiological evidence for silent inhibitory and excitatory connections, which represent a reserve pool that can be expressed following a period of activity. Both pre- and postsynaptic mechanisms are implicated in this work.

Electrophysiological experiments can demonstrate the existence of silent synapses indirectly by showing recruitment of initially silent synapses into the active state. Experiments in the mammalian spinal cord demonstrated silent synapses between group Ia afferents and motor neurons (Redman 1990). Statistical procedures were used to estimate the number of boutons from a single afferent contributing to the excitatory postsynaptic potential (EPSP). Broadening the presynaptic action potential, or repetitive stimulation, increased the number of contributing boutons. Anatomical reconstructions showed that the number of boutons available sometimes exceeded the number estimated statistically at low frequencies. Similar results have been obtained in tissue culture preparations (Pun et al. 1986), but the spinal cord evidence is of particular significance because it was obtained from an intact developed system. Statistical evidence, although suggestive, ultimately requires matched ultrastructural studies to demonstrate unequivocally the presence of silent synapses (as we have defined them).

Further experiments have been done extensively on a number of preparations from the mammalian CNS, but it should be noted that most of these preparations were obtained from relatively young animals, usually rats or mice, for technical reasons. For example, dentate granule neurons grown in cell culture form silent projections onto themselves (autapses). These synapses express both AMPA and NMDA receptors and one can conveniently study their properties using either NMDA or AMPA blockers. In a clever experiment, Tong et al. (1996) first blocked the AMPA receptors, and then used the open channel blocking properties of MK-801 to progressively and selectively block NMDA receptors at active synapses. After complete blockade, it was still possible to recruit new synapses by high-frequency stimulation (Fig. 8). Under normal conditions, these synapses form mossy fiber projections to CA3. Thus, the LTP at this synaptic projection appears to consist of recruitment of previously inactive synapses (Tong et al. 1996). The mechanism was evidently presynaptic because blockade of the postsynaptic receptors with Kynurenic acid did not block the LTP. It would be worthwhile to verify this result in the slice preparation to establish whether the 


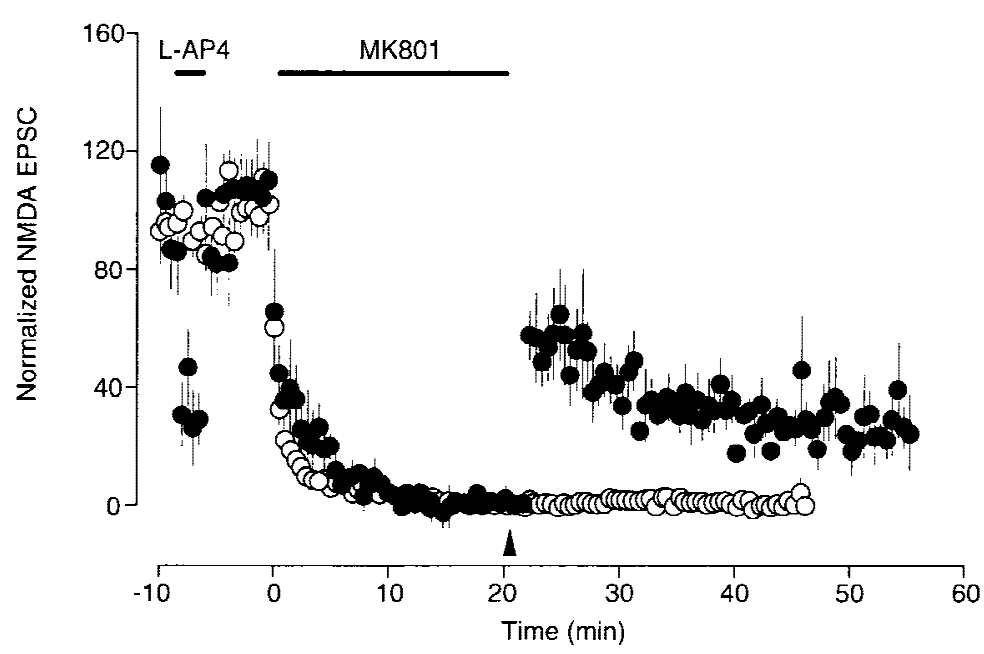

Figure 8: Unmasking of initially silent synapses by pharmacological treatment. In cultures of dentate granule cells, mossy fibers form autapses at which NMDA-dependent responses can be isolated in the presence of an AMPA receptor blocker (CNQX). EPSCs attributable to NMDA receptors were blocked by MK801 (an open channel blocker) but additional responses, presumably those that were silent, appear with tetanic stimulation (arrowhead) after initial responses were blocked. Application of L-AP4 to depress glutamate release confirmed the identity of mossy fiber autapses (from Tong et al. 1996).

activity-dependent up-regulation of presynaptic release sites is a normal property of the intact mossy fiber terminals in CA3.

Another mechanism for recruitment of inactive synapses in CA1 has been proposed by Bolshakov et al. (1997) who used a particularly difficult technique of recording from pairs of connected CA3 and CA1 pyramidal neurons in slices. In studies of synaptic transmission, this is the technique of choice because it eliminates the uncertainty associated with more common approaches in which many afferents are simultaneously activated by an extracellular stimulating electrode. Surprisingly, under control conditions, there appeared to be only one active synapse per pair of connected neurons, as identified by a single quantal unit. Silent connections could not be verified, because morphological analysis was not done. Nevertheless, on the induction of LTP by application of cAMP to the slice, there was a high incidence of pairs with multiple synaptic contacts. This result is consistent with the appearance of multiple synaptic contacts either through activation of previously silent synapses or possibly by the formation of new ones. One caveat of these results is the possibility that the cAMP-induced effect may not be the same as the electrically induced LTP; two different mechanisms could exist. A definite answer to this remaining problem will have to await the combined use of electrophysiology and electron microscopy.

An alternative approach for demonstrating recruitment of silent synapses is to label synapses with endocytotic markers such as FM1-43 or synaptotagmin (Betz and Bewick 1992). Completely silent synapses cannot be labeled by this method, because endocytosis would not occur; however, one could detect a population of synapses with very low probabilities that would be regarded as essentially silent. A study of cultured hippocampal neurons by Murthy et al. (1997) showed that the 
CORRELATION OF STRUCTURE AND FUNCTION

DE NOVO SPINE/

SYNAPSE FORMATION distribution of release probabilities determined by destaining of the FM1-43-loaded terminals was uniform, peaking at $P=0.2$, and showing no evidence of a significant silent population. In contrast, the presence of a silent synaptic population was revealed by Chavis et al. (1998), who used two types of anti-synaptotagmin antibodies to label vesicle recycling in cultured cerebellar neurons. One of the antibodies was raised in rabbit and tagged with Texas Red, and the other, raised in goat, was tagged with Fluorescein (green). Silent synapses were found through the appearance of green-only puncta after the induction of LTP by application of forskolin, an adenylate cyclase stimulator. Although this approach holds promise for further studies of synaptic recruitment, one cannot be certain if the fluorescent puncta are individual synapses or synaptic boutons. There is also the possibility that the labeled sites are formed by postsynaptic exocytosis, which could be mistaken for a presynaptic event.

Given the uncertainty arising from physiological experiments, the correlation of ultrastructure and function is the preferred method of detecting silent synapses and their possible active counterparts. In a limited number of studies, pairs of connected neurons have been investigated by electrophysiology and electron microscopy (EM). Results have been obtained for several pathways in the CNS. The connections between cortical pyramidal cells and inhibitory interneurons in the cat visual cortex all were found to be active with high reliability, and no evidence of silent connections was found (Buhl et al. 1997). Connections from the excitatory CA3 pyramidal cells in the hippocampus onto the inhibitory interneurons have also been examined. In this case, it was found that each axon forms a single, strong synapse on the interneuron (Gulyas et al. 1993). These connections were also active and no evidence of latent synaptic contact was found.

A study by Markram et al. (1997) correlating the synaptic strength of excitatory connections between pairs of cortical pyramidal cells in conjunction with anatomical reconstructions of synaptic boutons found no evidence of silent synaptic contacts. Such studies are complicated by technical problems due to the uncertainty of staining all axonal branches by intracellular markers such as biocytin, and by poor preservation of the histological material for detailed EM examination. Evidence that not all anatomical synaptic sites were seen in EM reconstructions allows for the possibility that silent synapses were present, but positive evidence is still lacking.

All studies done so far involving ultrastructure have examined synapses in local circuits. This choice made the studies easier due to the relatively short distance between the pairs of connected neurons. Connections on inhibitory interneurons have the added advantage of being located on or near the cell body, making the EM reconstructions more feasible.

However, additional studies of long-distance projections such as CA3-CA1 Schaffer collaterals or granule cell-CA3 mossy fibers will be needed to search for the presence of silent synapses, because in these projections, suggestive electrophysiological evidence for recruitment of such synapses is already available.

Given that new synapses are likely to be of frequent occurrence in some neural circuits (Fig. 4), they could exist as nontransmitting structural entities (Fig. 2) for variable periods of time, and appear physiologically in response to an activating stimulus, or during a genetically programmed 
developmental sequence. In the mammalian CNS, dendritic spines are the usual sites of excitatory synaptic contacts. The spine/synapse unit (Fig.

1C) appears to be very plastic and can be regulated by ongoing synaptic activity in both developing and mature neurons (for review, see Harris and Kater 1994). The new spines and synapses can be formed from available cellular components in a very short time (minutes). Thus, neurons appear to possess synapses in a kit form that can be assembled or disassembled as the need arises (Fig. 2).

Appearance of new spines and synapses has been reported in numerous in vivo studies after direct brain stimulation (Geinisman et al. 1993; Trommald et al. 1996) or after placing animals in a learning situation or novel environment (Jones et al. 1997; Andersen and Soleng 1998). In cell culture preparations, induction of spines has been seen after various manipulations of neuronal activity. For example, Murphy et al. (1998) reported that activation of estrogen receptors on inhibitory interneurons can result in the appearance of new spines on the excitatory principal neurons, presumably via reduction of the inhibitory input from the interneurons to the principal cells.

There is, at present confusing evidence for the induction of new spines in brain slices. Sorra and Harris (1998) found no evidence of changes in either spine or synapse number following LTP in the CA1 area of the hippocampus. Surprisingly though, the same group (Kirov et al. 1999) reported that the slicing procedure induces the formation of new spines and synapses, so that the slices have more synapses than intact tissue. The cause of this phenomenon has not been determined. Engert and Bonhoeffer (1999) have found that synaptic activation of neurons in explant cultures of the hippocampus can induce formation of new spines in parallel with the induction of LTP. Thus, it would appear that, at least in some experimental situations, new spines and synapses can be rapidly produced in response to enhanced synaptic input. This is a striking example of activation of additional new synapses (rather than pre-existing and previously inactive synapses), albeit in somewhat artificial conditions specific for tissue culture. We note that de novo formation of synapses differs from recruitment of silent synapses, as we have defined them. In practice, it is often difficult to distinguish which is occurring unless both physiological and structural evidence are obtained.

Taken as a whole, mammalian studies have provided many instances in which conversion of synapses with NMDA receptors to synapses with additional AMPA-type receptors has been documented, but most of these studies are on tissue-cultured or developing preparations. Few studies in the mature nervous system have found good evidence for this mechanism. Formation of new synapses has been well documented, but this is not the same as recruitment of already-formed silent synapses.

\section{Summary of Possible Mechanisms}

The evidence to date favors the existence of silent synapses in several neural systems, and suggests also that both pre- and postsynaptic mechanisms may create silent synapses, and lead to their switching to an active state. In the above discussion, we have mapped out some of the factors that could be responsible for silent synapses (Fig. 2), and we have emphasized that developmental factors may often influence the occurrence of silent or conditionally silent synapses (Figs. 4,5). Many possible mechanisms could account for the observed results. In essence,

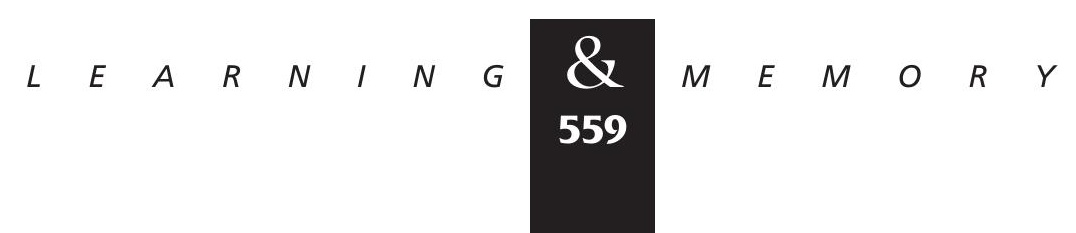


RAPID FORMATION, TURNOVER, AND REMODELING
RECEPTOR RECRUITMENT AND INSERTION recruitment of silent synapses, as we have defined it, depends on strengthening the synapses' physiological performance; thus, mechanisms that result in synaptic strengthening are candidates for the recruitment process. We will present a brief catalog of possible mechanisms, but without comprehensively reviewing all of the work on synaptic strength, and without detailed analysis of molecular substrates, which is beyond the scope of the present review.

That synapses can appear and also be turned over rapidly is well illustrated by ultrastructural work on insect eyes (Meinertzhagen 1993) and by physiological and structural observations in tissue cultures of mammalian (Haydon and Drapeau 1995) and invertebrate neurons (Coulson and Klein 1997). In some cases, synapses form and are functional in less than an hour, and do not require nuclear protein synthesis for functionality; the necessary components are already available, or can be locally synthesized. Such observations support a general theme that has been prevalent for many years in studies of the mammalian CNS: Synapse formation accompanies learning and related activities, even in the adult brain (Andersen and Soleng 1998). But it is not clear whether newly formed synapses qualify as recruitable silent synapses. The physiological attributes of newly formed synapses in the mammalian CNS have yet to be fully worked out; their availability as recruitable silent synapses is not well established. Moreover, remodeling of synapses (presumably enhancing the efficacy of the connection through increases in their size or number) has been described in the mammalian CNS (Greenough 1984; Desmond and Levy 1990; Edwards 1995) and after LTF in crustacean neurons (Wojtowicz et al. 1994). In the mature CNS, remodeling is a good candidate mechanism for recruitment of silent synapses.

Progressive turnover and remodeling of synapses is probably a frequent occurrence in many neurons. For example, in crustacean motor neurons (Fig. 5), synapses are lost and generated throughout life, and the many synapses lacking active zone dense bodies, which are likely silent, are probably transitional synapses, either incompletely established or in the process of being decommissioned. Thus, in general, silent synapses can arise as a natural consequence of growth or development. In such cases, they may respond rapidly to hormonal or activity-induced changes, as do conditionally silent synapses.

Important mechanisms underlying synapse formation and stabilization include (1) effects of neurotrophic factors such as NT-3 and BDNF, which have been shown to influence synaptic potency of developing neuromuscular junctions, possibly through activation of receptor tyrosine kinases (Lohof et al. 1993; Kwon and Gurney 1996); and (2) retrograde and anterograde signaling across the synapse, which affects its properties and may determine the frequency of silent synapses in each connection (DiAntonio et al. 1999; Wan and Poo 1999). These actively investigated mechanisms will provide additional information about determinants of synaptic strength and whether such mechanisms are relevant to recruitment of silent synapses.

As already discussed, a dominant hypothesis at present for synaptic recruitment at hippocampal glutamatergic synapses is the conversion of

$$
\begin{array}{lllllllllllllll}
\hline & E & A & R & N & I & N & G & \underset{560}{\mathcal{Z}} & M & E & M & O & R & Y
\end{array}
$$


TRANSMITTER SPILLOVER

SYNAPTIC PROTEINS AND VESICLE POOLS
NMDA receptor-containing (conditionally silent) synapses to AMPA receptor-containing synapses, a process that is particularly well seen in tissue culture preparations and in the developing vertebrate nervous system, but less easily observed in the mature nervous system. Rapid delivery of AMPA receptors to dendritic spines (Fig. 2E) following NMDA receptor activation probably mediates this transformation (Shi et al. 1999). In addition, the less well-studied kainate receptors undergo developmental and activity-dependent down-regulation in parts of the mammalian CNS, correlated with their decreasing contribution to neurotransmission (Kidd and Isaac 1999).

Glutamate receptor activation at the Drosopbila neuromuscular junction is thought to be responsible for a retrograde signal, which in turn influences the transmitter-releasing capabilities of the presynaptic terminal (DiAntonio et al. 1999). Retrograde signals have also been indicated in neonatal rat hippocampus (e.g., Caillard et al. 1999, for GABAergic synapses). In addition, glutamate receptor activation may effect morphological alterations (dendritic spine shape changes) affecting synaptic plasticity through interactions with cytoskeletal proteins (Van Rossum and Hanisch 1999). Thus, postsynaptic receptors have several roles in determination of synaptic properties.

Glycine spillover from silent to active synapses on the Mauthner cell furnishes an example of synaptic cross talk between silent and functional synapses (Faber et al. 1991). More recently, Kullmann and others have investigated cross talk among glutamatergic synapses in the mammalian CNS (Rusakov and Kullmann 1998; Rusakov et al. 1999). Glutamate spillover could, in principle, activate nearby synapses, perhaps recruiting some that otherwise would be silent (Malenka and Nicoll 1997). It is even possible that the NMDA receptor synapses are activated by spillover from adjacent synapses, because NMDA receptors have a lower $K_{D}$ for glutamate than AMPA receptors. However, whereas manifestations of the spillover effect are observable at experimental temperatures of $30^{\circ} \mathrm{C}$ and lower, it has not been established clearly that this effect is significant at brain temperature $\left(37^{\circ} \mathrm{C}\right)$, as uptake of glutamate may reduce or eliminate it.

A set of mechanisms that definitely contribute to synaptic strengthening and that could establish presynaptically silent synapses (Fig. 2B) includes various modifications and adjustments of key synaptic proteins (for review, see Turner et al. 1999), and of stages in preparation of synaptic vesicles for release. Variations in synaptic proteins, including isoforms of synaptotagmin with different $\mathrm{Ca}^{2+}$-binding affinities (Littleton et al. 1999), and differences in expression of calcium-binding regulatory proteins such as crustacean frequenin (H.L. Atwood, M. Msghina, H. Lindemeier, and O. Pongs, unpubl.) are correlated with the exocytotic efficacy of synaptic boutons or regions within a synapse. Recruitment of synaptic vesicles to the releasable pool by a maturation process involving Munc13-1 (a presynaptic phorbol ester receptor) determines whether or not nerve-evoked transmitter release can occur at glutamatergic (but not GABAergic) synapses (Augustin et al. 1999); thus, derangement or insufficiency of this intracellular receptor can create a silent synapse. The normal conditions that regulate the supply of these and other presynaptic 
CALCIUM CHANNELS

CAMP AND OTHER SECOND MESSENGER SYSTEMS proteins are not well understood, but they provide potential candidates for explaining some cases of silent synapses.

Localization of different calcium channel isoforms in the mammalian CNS has been extensively reviewed and discussed (Catterall 1995). Clearly, the patterns of localization are functionally significant in synaptic transmission, and change during development (Scholz and Miller 1995). Of particular relevance for the present review is the following question: Can properties of the presynaptic calcium channels determine whether the synapse is silent or active? As yet, definite answers are lacking. Some forms of rapid depression appear to involve curtailment of calcium entry (Bellingham and Walmsley 1999), inactivation of intracellular receptors by $\mathrm{Ca}^{2+}$ (Hsu et al. 1996), or calcium channel inactivation (Forsythe et al. 1998). Such mechanisms could provide a possible explanation for a rapid increase in silent synapses during short-term depression (Fig. 7).

On the other hand, recent evidence from the crustacean neuromuscular junction (Msghina et al. 1999) suggests that lack of calcium entry is not the prime factor responsible for silent synapses on tonic neurons; instead, the calcium sensitivity of the vesicle-releasing mechanism is likely to be responsible. Calcium entry estimated per active zone in tonic varicosities suggests fully functional $\mathrm{Ca}^{2+}$ channels (Tank et al. 1995). Transmitter release per active zone is 100-1000 times larger for synapses of phasic motor axons than in tonic motor axons, yet calcium entry per active zone is about the same. Thus, calcium channel properties may not be primarily responsible for silent synapses in the tonic motor neurons. Calcium sensitivity of proteins involved in transmitter release appears to be regulated in many different synaptic systems by presynaptic effects of neurotransmitters and neuromodulators (Yawo 1996).

cAMP, through its activation of protein kinase A (PKA) and consequent phosphorylation of a variety of intracellular protein substrates, is known to enhance synaptic transmission by increasing transmitter release (Chavez-Noriega and Stevens 1994; Trudeau et al. 1996). Most of the effects attributable to elevated cAMP are presynaptic. In crayfish motor neurons, LTF is abolished when generation of cAMP or activation of PKA is prevented (Dixon and Atwood 1989). Elevation of cAMP induces vesicle cycling at previously silent sites of cultured cerebellar granule cell synapses (Chavis et al. 1998), and this could be involved in cerebellar LTP. Various presynaptic receptors are known to influence cAMP production, and could thus, in principle, recruit silent synapses.

Other second messenger systems also modulate synaptic transmitter release or postsynaptic properties. For example, in the calyciform synapse of the chick ciliary ganglion, pharmacological stimulation of protein kinase $\mathrm{C}$ enhances transmitter release by up-regulating the releasable pool of synaptic vesicles or the number of release sites (Yawo 1999).

$\mathrm{Ca}^{2+} /$ calmodulin-dependent protein kinase II regulates clustering of postsynaptic molecules (Koh et al. 1999). This enzyme can be produced by dendritic protein synthesis and has been shown to increase postsynaptically in dendrites following tetanic stimulation (Ouyang et al. $1997,1999)$. These effects form a basis for postsynaptic activity-dependent modification.

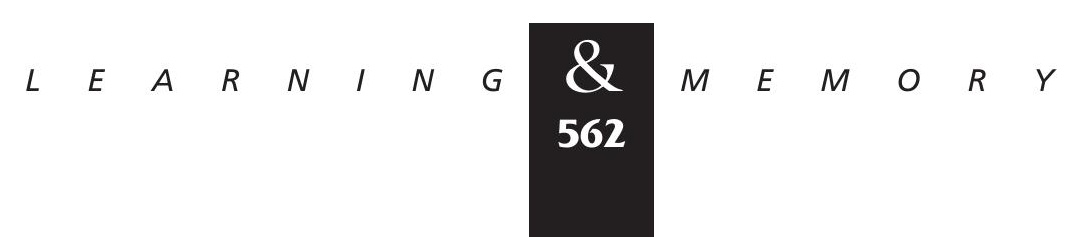


Functional

SHORT-TERM FACILITATION AND DEPRESSION

LTP AND CIRCUIT MODIFICATION
Evidence has been provided that silent synapses are recruited to activity during frequency facilitation. Conversely, synapses may become silent during low-frequency depression. Functionally, these effects provide a mechanism for rapid expansion or contraction of neural circuits. Phenomena such as rapid activity-dependent expansion of receptive fields (Wall 1977) could use these mechanisms. In fact, a recent study has shown that sensory experience modifies short-term synaptic dynamics in excitatory pathways of the cortex; these modifications may influence functional properties of cortical circuits resulting from repeated sensory experience (Finnerty et al. 1999), and could make use of silent synapse recruitment.

The original suggestion of Bliss and Lomo (1973) that LTP may serve as an information storage mechanism was, and still is, an attractive hypothesis. Several features of LTP, notably its associative induction and long-lasting duration, make it the most likely mechanisms for information storage in the hippocampus and elsewhere in the brain. However, numerous attempts to pin down the exact relationship between LTP and the behavioral phenomenon of learning have been inconclusive. Several recent studies have supported a correlation between enhanced potentiation and improved learning (Tang et al. 1999). However, along with many studies showing suggestive correlative evidence for such a relationship, there are others that show a clear dissociation between the phenomena of LTP and learning. Particularly convincing is a double dissociation offered by studies of transgenic mice. In one such study, Motro et al. (1996) found that mice deficient in a ligand for Kit receptor tyrosine kinase (Steel factor) are impaired in learning but not in LTP. In another, mice deficient in the AMPA-type glutamate receptors were deficient in LTP but not in learning (Zamanillo et al. 1999). A factor contributing to this dilemma is the use of arbitrary and physiologically questionable protocols for LTP induction. In many such studies, brief trains of evenly spaced stimuli (often called tetanic stimulation) are used to activate the afferents of neurons under study. In others, a steady postsynaptic depolarization is applied by artificial means and paired with low frequency stimulation. Both experimental protocols have been effective in inducing LTP that has a mechanism consistent with the activation of previously silent synapses (Isaac et al. 1995; Liao et al. 1995; Wang et al. 1996). However, their physiological relevance in relation to learning remains to be explained.

Although the relevance of LTP for learning remains at present controversial and debatable, a clear link occurs between LTP and synaptic development. Particularly in the vertebrate CNS, the developing neurons of immature animals appear to possess a significant number of synapses endowed predominantly with NMDA receptors that, during maturation, are supplemented with AMPA receptors (Durand et al. 1996; Wu et al. 1996; Isaac et al. 1997; Golshani and Jones 1999). As a result, conditionally silent synapses become functional over a wider range of conditions and can presumably transmit more information from other parts of the nervous system. Activation of these synapses during 


\section{Acknowledgments}

\section{References}


Betz, W.J. and G.S. Bewick. 1992. Optical analysis of synaptic vesicle recycling at the frog neuromuscular junction. Science 255: 200-203.

Betz, W.J., F. Mao, and G.S. Bewick. 1992. Activity-dependent fluorescent staining and destaining of living vertebrate motor nerve terminals. J. Neurosci. 12: 363-375.

Bliss, T.V.P. and T. Lomo. 1973. Long-lasting potentiation of synaptic transmission in the dentate area of the anaesthetized rabbit following stimulation of the perforant path. J. Physiol. 232: 331-356.

Bolshakov, V.Y., H. Golan, E.R. Kandel, and S.A. Siegelbaum. 1997. Recruitment of new sites of synaptic transmission during the cAMP-dependent late phase of LTP at CA3-CA1 synapses in the hippocampus. Neuron 19: 635-651.

Bommert, K., M.P. Charlton, W.M. DeBello, G.J. Chin, H. Betz, and G.J. Augustine. 1993. Inhibition of neurotransmitter release by C2-domain peptides implicates synaptotagmin in exocytosis. Nature 363: 163-165.

Brain, K.L., L.J. Cottee, and M.R. Bennett. 1997. Varicosities of single sympathetic nerve terminals possess syntaxin zones and different synaptotagmin N-terminus labelling following stimulation. J. Neurocytol. 26: 491-500.

Bruner, J. and D. Kennedy. 1970. Habituation: Occurrence at a neuromuscular junction. Science 169: 92-94.

Buhl, E.H., T. Szilágyi, C. Stricker, O. Paulsen, and P. Somogyi. 1997. Effect, number and location of synapses made by single pyramidal cells onto spiny interneurones of cat visual cortex. J. Physiol. 500.3: 689-713.

Caillard, O., Y. Ben-Ari, and J.-L. Gaiarsa. 1999. Long-term potentiation of GABAergic synaptic transmission in neonatal rat hippocampus. J. Physiol. 518.1: 109-119.

Catterall, W.A. 1995. Structure and function of voltage-gated ion channels. Annu. Rev. Biochem. 64: 493-531.

Charpier, S., J.C. Behrends, A. Triller, D.S. Faber, and H. Korn. 1995. "Latent" inhibitory connections become functional during activity-dependent plasticity. Proc. Natl. Acad. Sci. 92: $117-120$.

Chavez-Noriega, L.E. and C.F. Stevens. 1994. Increased transmitter release at excitatory synapses produced by direct activation of adenylate cyclase in rat hippocampal slices. J. Neurosci. 14: 310-317.

Chavis, P., P. Mollard, J. Bockaert, and O. Manzoni. 1998. Visualization of cyclic AMP-regulated presynaptic activity at cerebellar granule cells. Neuron 20: 773-781.

Cooper, R.L., L. Marin, and H.L. Atwood. 1995. Synaptic differentiation of a single motor neuron: Conjoint definition of transmitter release, presynaptic calcium signals, and ultrastructure. J. Neurosci. 15: 4209-4222.

Cooper, R.L., J.L. Winslow, C.K. Govind, and H.L. Atwood. 1996. Synaptic structural complexity as a factor enhancing probability of calcium-mediated transmitter release. J. Neurophysiol. 75: 2451-2466.

Coulson, R.L. and M. Klein. 1997. Rapid development of synaptic connections and plasticity between sensory neurons and motor neurons of Aplysia in cell culture: Implications for learning and regulation of synaptic strength. J. Neurophysiol. 77: 2316-2327.

Couteaux, R. 1970. Vesicles synaptiques et poches au niveau des "zones active" de la junction neuromusculaire. C. R. Acad. Sci. Ser. D 271: 2346-2349.

del Castillo, J. and B. Katz. 1956. Localization of active spots within the neuromuscular junction of the frog. J. Physiol. 132: 630-649.

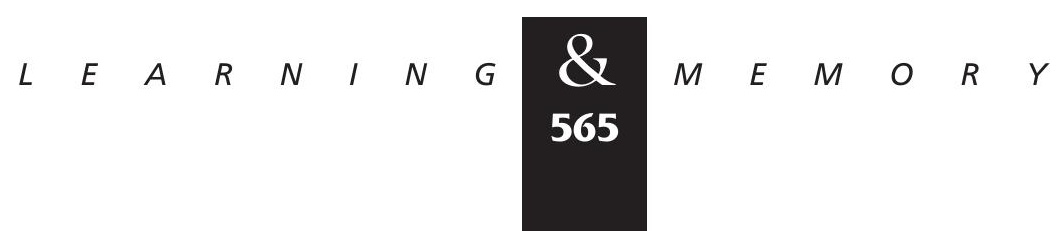


Desmond, N.L. and W.B. Levy. 1990. Morphological correlates of long-term potentiation imply the modification of existing synapses, not synaptogenesis, in the hippocampal dentate gyrus. Synapse 5: 139-143.

DiAntonio, A., S.A. Petersen, M. Heckmann, and C.S. Goodman. 1999. Glutamate receptor expression regulates quantal size and quantal content at the Drosophila neuromuscular junction. J. Neurosci. 19: 3023-3032.

Dixon, D. and H.L. Atwood. 1989. Adenylate cyclase system is essential for long-term facilitation at the crayfish neuromuscular junction. J. Neurosci. 9: 4246-4252.

Durand, G.M., Y. Kovalchuk, and A. Konnerth. 1996. Long-term potentiation and functional synapse induction in developing hippocampus. Nature 381: 71-75.

Edwards, F.A. 1995. Anatomy and electrophysiology of fast central synapses lead to a structural model for long-term potentiation. Physiol. Rev. 75: 759-787.

Engert, F. and T. Bonhoeffer. 1999. Dendritic spine changes associated with hippocampal long-term synaptic plasticity. Nature 399: 66-70.

Faber, D.S., J.-W. Lin, and H. Korn. 1991. Silent synaptic connections and their modifiability. Ann. N.Y. Acad. Sci. 627: 151-164.

Feldman, D.R., R.A. Nicoll, and R.C. Malenka. 1999. Synaptic plasticity at thalamocortical synapses in developing rat somatosensory cortex: LTP, LTD, and silent synapses. J. Neurobiol. 41: 92-101.

Finnerty, G.T., L.S.E. Roberts, and B.W. Connors. 1999. Sensory experience modifies the short-term dynamics of neocortical synapses. Nature 400: 367-371.

Forsythe, I.D., T. Tsujimoto, M. Barnes-Davies, M.F. Cuttle, and T. Takahashi. 1998. Inactivation of presynaptic calcium current contributes to synaptic depression at a fast central synapse. Neuron 20: 797-807.

Franzini-Armstrong, C. 1976. Freeze-fracture of excitatory and inhibitory synapses in crayfish neuromuscular junctions. J. Microsc. Biol. Cell. 25: 217-222.

Geinisman, Y., L. DeToledo-Morrell, F. Morrell, R.E. Heller, M. Rossi, and R.F. Parshall. 1993. Structural synaptic correlate of long-term potentiation: Formation of axospinous synapses with multiple, completely partitioned transmission zones. Hippocampus 3: $435-446$.

Golshani, P. and E.G. Jones. 1999. Synchronized paroxysmal activity in the developing thalamocortical network mediated by corticothalamic projections and "silent" synapses. J. Neurosci. 19: 2865-2975.

Govind, C.K., H.L. Atwood, and J. Pearce. 1995. Inhibitory axoaxonal and neuromuscular synapses in the crayfish opener muscle: Membrane definition and ultrastructure. J. Comp. Neurol. 351: 476-488.

Greenough, W.T. 1984. Structural correlates of information storage in the mammalian brain: A review and hypothesis. Trends Neurosci. 7: 229-233.

Gulyas, A.I., R. Miles, N. Hajos, and T.F. Freund. 1993. Precision and variability in postsynaptic target selection of inhibitory cells in the hippocampal CA3 region. Eur. J. Neurosci. 5: 1729-1751.

Harris, K.M. and S.B. Kater. 1994. Dendritic spines: Cellular specializations imparting both stability and flexibility of synaptic function. Annu. Rev. Neurosci. 17: 341-371.

Haydon, P.G. 1988. The formation of chemical synapses between cell-cultured neuronal somata. J. Neurosci. 8: 1032-1038.

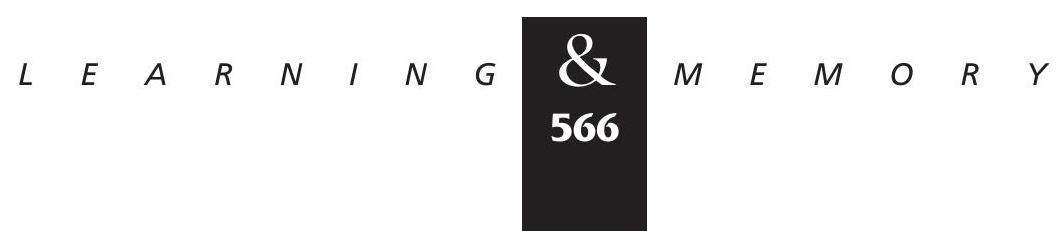


Haydon, P.G. and P. Drapeau. 1995. From contact to connection: Early events during synaptogenesis. Trends Neurosci. 18: 196-201.

He, Y., W.G.M. Janssen, and J.H. Morrison. 1998. Synaptic coexistence of AMPA and NMDA receptors in the rat hippocampus: A postembedding immunogold study. J. Neurosci. Res. 54: 444-449.

Hebb, D.O. 1949. The organization of behavior. Wiley, New York, NY.

Hsu, S.-F., G.J. Augustine, and M.B. Jackson. 1996. Adaptation of $\mathrm{Ca}^{2+}$-triggered exocytosis in presynaptic terminals. Neuron 17: 501-512.

Isaac, J.T.R., R.A. Nicoll, and R.C. Malenka. 1995. Evidence for silent synapses: Implications for the expression of LTP. Neuron 15: 427-434.

Isaac, J.T.R., M.C. Crair, R.A. Nicoll, and R.C. Malenka. 1997. Silent synapses during development of thalamocortical inputs. Neuron 18: 269-280.

Jahromi, S.S. and H.L. Atwood. 1974. Three-dimensional ultrastructure of the crayfish neuromuscular apparatus. J. Cell Biol. 63: 599-613.

Johnson, E.W. and A. Wernig. 1971. The binomial nature of transmitter release at the crayfish neuromuscular junction. J. Physiol. 281: 757-767.

Jones, T.A., A.Y. Klintsova, V.L. Kilman, A.M. Sirevaag, and W.T. Greenough. 1997. Induction of multiple synapses by experience in the visual cortex of adult rats. Neurobiol. Learn. Mem. 68: 13-20.

Kandel, E.R. and J.H. Schwartz. 1982. Molecular biology of learning: Modulation of transmitter release. Science 218: 433-443.

Kidd, F.L. and J.T.R. Isaac. 1999. Developmental and activity-dependent regulation of kainate receptors at thalamocortical synapses. Nature 400: 569-573.

Kirov, S.A., K.E. Sorra, and K.M. Harris. 1999. Slices have more synapses than perfusion-fixed hippocampus from both young and mature rats. J. Neurosci. 19: 2876-2886.

Koh, Y.H., E. Popova, U. Thomas, L.C. Griffith, and V. Budnik. 1999. Regulation of DLG localization at synapses by CaMKII-dependent phosphorylation. Cell 98: 353-363.

Korn, H. 1998. Morphological principles. In Central synapses: Quantal mechanisms and plasticity (ed. D.S. Faber, H. Korn, S.J. Redman, S.M. Thompson, and J.S. Altman), pp. 19-23. Human Frontier Science Program, Strasbourg, France.

Kullmann, D.M. and R.A. Nicoll. 1992. Long-term potentiation is associated with increases in quantal content and quantal amplitude. Nature 357: 240-244.

Kwon, Y.W. and M.E. Gurney. 1996. Brain-derived neurotrophic factor transiently stabilizes silent synapses on developing neuromuscular junctions. J. Neurobiol. 29: 503-516.

Lavidis, N.A. and M.R. Bennett. 1992. Probabilistic secretion of quanta from visualized sympathetic nerve varicosities in mouse vas deferens. J. Physiol. (Lond.) 454: 9-26.

Liao, D., N.A. Hessler, and R. Malinow. 1995. Activation of postsynaptically silent synapses during pairing-induced LTP in CA1 region of hippocampal slice. Nature 375: 400-404.

Littleton, J.T., M. Stern, K. Schulze, M. Perin, and H.J. Bellen. 1993. Mutational analysis of Drosophila synaptotagmin demonstrates its essential role in $\mathrm{Ca}^{2+}$-activated neurotransmitter release. Cell 74: 1125-1134.

Littleton, J.T., T.L. Serano, G.M. Rubin, B. Ganetzky, and E.R. Chapman. 1999. Synaptic function modulated by changes in the ratio of synaptotagmin I and IV. Nature

400: $757-780$.

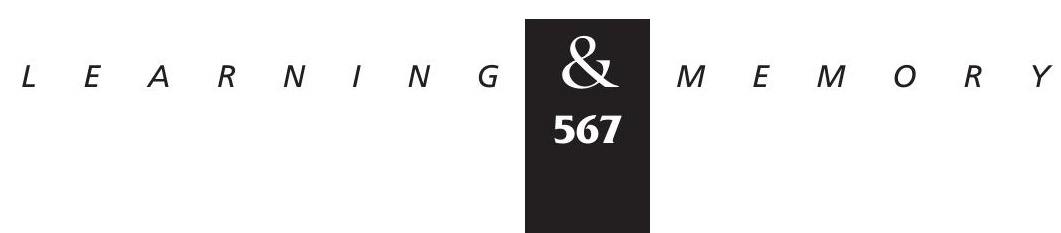


Lohof, A.M., N.Y. Op, and M.-M. Poo. 1993. Potentiation of developing neuromuscular synapses by the neurotrophins NT-3 and BDNF. Nature 363: 350-353.

Macleod, G.T., J.-B. Gan, and M.R. Bennett. 1999. Vesicle-associated proteins and quantal release at single active zones of amphibian (Bufo marinus) motor-nerve terminals. J. Neurophysiol. 82: 1133-1146.

Malenka, R. 1998. Silent synapses in the hippocampus and cortex. In Central synapses: Quantal mechanisms and plasticity (ed. D.S. Faber, H. Korn, S.J. Redman, S.M. Thompson, and J.S. Altman), pp. 207-214. Human Frontier Science Program, Strasbourg, France.

Malenka, R.C. and R.A. Nicoll. 1997. Silent synapses speak up. Neuron 19: 473-476. 1999. Long-term potentiation—A decade of progress? Science 285: 1870-1874.

Malinow, R. 1998. Silent synapses in three forms of central plasticity. In Central synapses: Quantal mechanisms and plasticity (ed. D.S. Faber, H. Korn, S.J. Redman, S.M. Thompson, and J.S. Altman), pp. 226-234. Human Frontier Science Program, Strasbourg, France.

Markram, H., J. Lübke, M. Frotscher, A. Roth, and B. Sakmann. 1997. Physiology and anatomy of synaptic connections between thick tufted pyramidal neurones in the developing rat neocortex. J. Physiol. (Lond. ) 500.2: 409-440.

Meinertzhagen, I.A. 1993. The synaptic populations of the fly's optic neuropil and their dynamic regulation: Parallels with the vertebrate retina. Prog. Retinal Res. 12: 13-39.

Motro, B., J.M. Wojtowicz, A. Bernstein, and D. Van der Kooy. 1996. "Steel" mutant mice are deficient in hippocampal learning but not long-term potentiation. Proc. Natl. Acad. Sci. 93: 1808-1813.

Msghina, M., A.G. Millar, M.P. Charlton, C.K. Govind, and H.L. Atwood. 1999. Calcium entry related to active zones and differences in transmitter release at phasic and tonic synapses. J. Neurosci. 19: 8419-8434.

Murphy, D.D., N.B. Cole, V. Greenberger, and M. Segal. 1998. Estradiol increases dendritic spine density by reducing GABA neurotransmission in hippocampal neurons. J. Neurosci. 18: $2550-2559$.

Murthy, V.N., T.J. Sejnowski, and C.F. Stevens. 1997. Heterogeneous release properties of visualized individual hippocampal synapses. Neuron 18: 599-612.

Nusser, Z., R. Lujan, G. Laube, J.D.B. Roberts, E. Molńr, and P. Somogyi. 1998. Cell type and pathway dependence of synaptic AMPA receptor number and variability in the hippocampus. Neuron 21: 545-559.

Ouyang, Y., D. Kantor, K.M. Harris, E.M. Schuman, and M.B. Kennedy. 1997. Visualization of the distribution of autophosphorylated calcium/ calmodulin-dependent protein kinase II after tetanic stimulation in the CA1 area of the hippocampus. J. Neurosci. 17: 5416-5427.

Ouyang, Y., A. Rosenstein, G. Kreiman, E.M. Schuman, and M.B. Kennedy. 1999. Tetanic stimulation leads to increased accumulation of $\mathrm{Ca}^{2+} /$ calmodulin-dependent protein kinase II via dendritic protein synthesis in hippocampal neurons. J. Neurosci. 19: 7823-7833.

Pawson, P.A., A.D. Grinnell, and B. Wolowske. 1998. Quantitative freeze-fracture analysis of the frog neuromuscular junction synapse - I. Naturally occurring variability in active zone structure. J. Neurocytol. 27: 361-377.

Pun, R.Y., E.A. Neale, P.B. Guthrie, and P.G. Nelson. 1986. Active and inactive central synapses in cell culture. J. Neurophysiol. 56: 1242-1256.

Quigley, P.A., M. Msghina, C.K. Govind, and H.L. Atwood. 1999. Visible evidence for differences in synaptic effectiveness with activity-dependent vesicular uptake and release of FM1-43. J. Neurophysiol. 81: 356-370. 
Redman, S. 1990. Quantal analysis of synaptic potentials in neurons of the central nervous system. Physiol. Rev. 70: 165-198.

Royer, S., R.L. Coulson, and M. Klein. 1999. Switching off and on of synaptic sites at Aplysia sensorimotor synapses. J. Neurosci. (In press).

Rumpel, S., H. Hatt, and K. Gottmann. 1998. Silent synapses in the developing rat visual cortex: Evidence for postsynaptic expression of synaptic plasticity. J. Neurosci.

18: $8863-8874$.

Rusakov, D.A. and D.M. Kullmann. 1998. Extrasynaptic glutamate diffusion in the hippocampus: Ultrastructural constraints, uptake, and receptor activation. J. Neurosci. 18: $3158-3170$.

Rusakov, D.A., D.M. Kullman, and M.G. Stewart. 1999. Hippocampal synapses: Do they talk to their neighbours? Trends Neurosci. 22: 382-388.

Scholz, K.P. and R.J. Miller. 1995. Developmental changes in presynaptic calcium channels coupled to glutamate release in cultured rat hippocampal neurons. J. Neurosci. 15: 4612-4617.

Sherman, R.G. and H.L. Atwood. 1971. Synaptic facilitation: Long-term neuromuscular facilitation in crustaceans. Science 171: 1248-1250.

Shi, S.H., Y. Hayashi, R.S. Petralia, S.H. Zaman, R.J. Wenthold, K. Svoboda, and R. Malinow. 1999. Rapid spine delivery and redistribution of AMPA receptors after synaptic NMDA receptor activation. Science 284: 1811-1816.

Smith, B.R., J.M. Wojtowicz, and H.L. Atwood. 1991. Maximum likelihood estimation of non-uniform transmitter release probabilities at the crayfish neuromuscular junction. J. Theor. Biol. 150: 457-472.

Sorra, K.E. and K.M. Harris. 1998. Stability in synapse number and size at $2 \mathrm{hr}$ after long-term potentiation in hippocampal area CA1. J. Neurosci. 18: 658-671.

Stevens, C.F. and Y. Wang. 1994. Changes in reliability of synaptic function as a mechanism for plasticity. Nature 371: 704-707.

Stricker, S., S.J. Redman, A.C. Field, and S.P. Perrett. 1998. Statistical analysis of evoked EPSCs before and after the induction of LTP and LTD. In Central synapses: Quantal mechanisms and plasticity (ed. D.S. Faber, H. Korn, S.J. Redman, S.M. Thompson, and J.S. Altman), pp. 198-207. Human Frontier Science Program, Strasbourg, France.

Tang, Y.-P., E. Shimizu, G.R. Dube, C. Rampon, G.A. Kerchner, M. Zhuo, G. Liu, and J.Z. Tsien. 1999. Genetic enhancement of learning and memory in mice. Nature 401: 63-69.

Tank, D.W., W.G. Regehr, and K.R. Delaney. 1995. A quantitative analysis of presynaptic calcium dynamics that contribute to short-term enhancement. J. Neurosci. 15: 7940-7952.

Tong, G., R.C. Malenka, and R.A. Nicoll. 1996. Long-term potentiation in cultures of single hippocampal granule cells. Neuron 16: 1147-1157.

Trommald, M., G. Hulleberg, and P. Andersen. 1996. LTP is associated with new excitatory spine synapses on rat dentate granule cells. Learn. \& Mem. 3: 218-228.

Trudeau, L.-E., D.G. Emery, and P.G. Haydon. 1996. Direct modulation of the secretory machinery underlies PKA-dependent synaptic facilitation in hippocampal neurons. Neuron 17: 789-797.

Turner, K.M. and R.D. Burgoyne. 1999. Protein phosphorylation and the regulation of synaptic membrane traffic. Trends Neurosci. 22: 459-464.

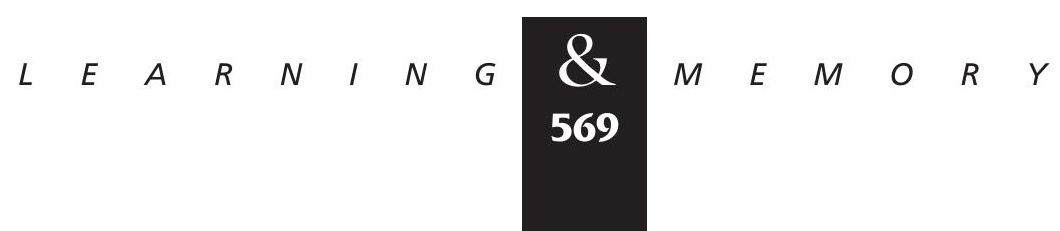


Turrigiano, G.G. 1999. Homeostatic plasticity in neuronal networks: The more things change, the more they stay the same. Trends Neurosci. 22: 221-227.

Umbach, J.A., K.E. Zinsmaier, K.K. Eberle, E. Buchner, S. Benzer, and C.B. Gundersen. 1994. Presynaptic dysfunction in Drosophila csp mutants. Neuron 13: 899-907.

Van Rossum, D. and U.-K. Hanisch. 1999. Cytoskeletal dynamics in dendritic spines: Direct modulation by glutamate receptors? Trends Neurosci. 22: 290-295.

Verheugen, J.A.H., D. Fricker, and R. Miles. 1999. Noninvasive measurements of the membrane potential and GABAergic action in hippocampal interneurons. J. Neurosci. 19: $2546-2555$.

Wall, P.D. 1977. The presence of ineffective synapses and the circumstances which unmask them. Phil. Trans. R. Soc. London. Ser. B 278: 361-372.

Wan, J.-J. and M.-M. Poo. 1999. Activity-induced potentiation of developing neuromuscular synapses. Science 285: 1725-1728.

Wang, C. and R.S. Zucker. 1998. Regulation of synaptic vesicle recycling by calcium and serotonin. Neuron 21: 155-167.

Wang, S., J.M. Wojtowicz, and H.L. Atwood. 1996. Synaptic recruitment during long-term potentiation at synapses of the medial perforant pathway in the dentate gyrus of the rat brain. Synapse 21: 78-86.

Wang, S., B.W. Scott, and J.M. Wojtowicz. 2000. Heterogenous properties of dentate granule neurons in the adult rat. J. Neurobiol. (In press).

Wernig, A. 1976. Localization of active sites in the neuromuscular junction of the frog. Brain. Res. 118: 63-72.

Wojtowicz, J.M. 1998. Regional differentiation of dendritic morphology and synaptic properties of dentate granule cells. In Central synapses: Quantal mechanisms and plasticity (ed. D.S. Faber, H. Korn, S.J. Redman, S.M. Thompson, and J.S. Altman), pp. 235-226. Human Frontier Science Program, Strasbourg, France.

Wojtowicz, J.M. and H.L. Atwood. 1985. Correlation of presynaptic and postsynaptic events during establishment of long term facilitation at the crayfish neuromuscular junction. J. Neurophysiol. 54: 220-230.

Wojtowicz, J.M., B.R. Smith, and H.L. Atwood. 1991. Activity-dependent recruitment of silent synapses. Ann. N.Y. Acad. Sci. 627: 169-179.

Wojtowicz, J.M., L. Marin, and H.L. Atwood. 1994. Activity-induced changes in synaptic release sites at the crayfish neuromuscular junction. J. Neurosci. 14: 3688-3702.

Worden, M.K., M. Bykhovskaia, and J.T. Hackett. 1997. Facilitation at the lobster neuromuscular junction: A stimulus-dependent mobilization model. J. Neurophysiol. 78: $417-428$.

Wu, G.-Y., R. Malinow, and H.T. Cline. 1996. Maturation of a central glutamatergic synapse. Science 274: 972-976.

Wu, M.N., T. Fergestad, T.E. Lloyd, Y. He, K. Broadie, and H.J. Bellen. 1999. Syntaxin-1A interacts with multiple exocytic proteins to regulate neurotransmitter release in vivo. Neuron 23: 593-605.

Yawo, H. 1996. Noradrenaline modulates transmitter release by enhancing the $\mathrm{Ca}^{2+}$ sensitivity of exocytosis in the chick ciliary presynaptic terminal. J. Physiol. (Lond.) 493: 385-391. 
1999. Protein kinase C potentiates transmitter release from the chick ciliary presynaptic terminal by increasing the exocytotic fusion probability. J. Physiol. 515.1: 169-180.

Zamanillo, D., R. Sprengel, O. Hvalby, V. Jensen, N. Burnashev, A. Rozov, K.M.M. Kaiser, H.J. Koster, T. Borchardt, P. Worley et al. 1999. Importance of AMPA receptors for hippocampal synaptic plasticity but not for spatial learning. Science 284: 1805-1811.

Zucker, R.S. 1973. Changes in the statistics of transmitter release during facilitation. J. Physiol. 229: 787-810. 


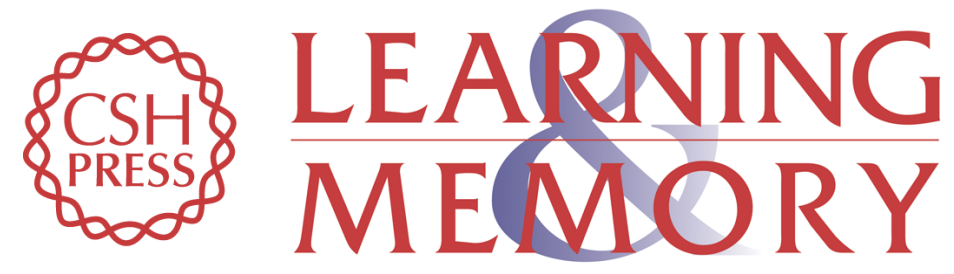

\section{Silent Synapses in Neural Plasticity: Current Evidence}

Harold L. Atwood and J. Martin Wojtowicz

Learn. Mem. 1999, 6:

References This article cites 111 articles, 34 of which can be accessed free at: http://learnmem.cshlp.org/content/6/6/542.full.html\#ref-list-1

License

Email Alerting Receive free email alerts when new articles cite this article - sign up in the box at the Service top right corner of the article or click here. 